\title{
Risk of acute stroke in patients with retinal artery occlusion: a systematic review and meta-analysis
}

\author{
Matteo Fallico $\mathbb{D}^{1,2} \cdot$ Andrew J. Lotery $\mathbb{B}^{2,3} \cdot$ Antonio Longo $^{1} \cdot$ Teresio Avitabile $^{1} \cdot$ Vincenza Bonfiglio ${ }^{1} \cdot$ \\ Andrea Russo ${ }^{1} \cdot$ Paolo Murabito $^{4} \cdot$ Stefano Palmucci $^{5} \cdot$ Alfredo Pulvirenti $^{6} \cdot$ Michele Reibaldi $^{1}$
}

Received: 11 June 2019 / Revised: 2 July 2019 / Accepted: 29 July 2019 / Published online: 16 September 2019

(c) The Author(s), under exclusive licence to The Royal College of Ophthalmologists 2019

\begin{abstract}
Objective To estimate the incidence of acute cerebral ischaemia detected by magnetic resonance imaging (MRI) in acute central retinal artery occlusion (CRAO), branch retinal artery occlusion (BRAO) and transient monocular vision loss (TMVL).

Methods Studies reporting the incidence of acute cerebral ischaemia, detected by MRI, within 7 days from diagnosis of acute CRAO, BRAO and TMVL up to January 2019 were systematically searched for on Pubmed, Medline and Cochrane Library. Meta-analysis was performed using random effects model. The primary outcome was the pooled estimate of incidence of acute cerebral ischaemia in CRAO, BRAO and TMVL cohorts including both neurologically symptomatic and asymptomatic patients, expressed as a proportion along with $95 \%$ confidence intervals (CIs). The pooled estimate of incidence of asymptomatic acute cerebral ischaemia represented a secondary outcome measure.

Results For the primary outcome, the pooled proportion of acute cerebral ischaemia was 0.30 (CI 0.24-0.36) in the CRAO cohort, and 0.25 (CI 0.16-0.37) in the BRAO cohort, without statistical heterogeneity. The rate of acute cerebral ischaemia was $11.8 \%$ in the TMVL cohort. For the secondary outcome, the pooled proportion of asymptomatic acute cerebral ischaemia was 0.22 (CI 0.16-0.28) in the CRAO cohort, 0.29 (CI 0.20-0.41) in the BRAO cohort and 0.08 (CI 0.05-0.15) in the TMVL cohort, with no statistical heterogeneity.

Conclusions $30 \%$ of patients with acute CRAO and $25 \%$ of patients with acute BRAO presented an acute cerebral ischaemia on MRI. Such high rates support a care pathway of prompt referral of such patients for neurological evaluation and brain imaging.
\end{abstract}

Supplementary information The online version of this article (https:// doi.org/10.1038/s41433-019-0576-y) contains supplementary material, which is available to authorized users.

Matteo Fallico

matteo.fallico@uhs.nhs.uk

1 Department of Ophthalmology, University of Catania, Catania, Italy

2 Eye Unit, Southampton University Hospital, Southampton, UK

3 Faculty of Medicine, University of Southampton, Southampton, UK

4 Department of Anaesthesiology, University of Catania, Catania, Italy

5 Department of Medical Surgical Sciences and Advanced Technologies - Radiology I Unit, University of Catania, Catania, Italy

6 Department of Clinical and Experimental Medicine, University of Catania, Catania, Italy

\section{Introduction}

Monocular vision loss (MVL) of ischaemic origin includes three different vascular retinal diseases: central retinal artery occlusion (CRAO), branch retinal artery occlusion (BRAO) and transient monocular visual loss (TMVL). These diseases differ in the size of the affected vessel and duration of occlusion [1]. CRAO and BRAO have been considered comparable with an ischaemic cerebral infarction, whilst TMVL is analogous to a transient ischaemic attack [2]. Furthermore, an increased risk of acute brain ischaemia has been seen in patients with retinal artery occlusion, the highest rate being within 7 days from diagnosis [3].

The issue as to whether patients with an acute retinal artery occlusion require urgent referral to the emergency department or stroke unit for neurologic evaluation is unresolved $[4,5]$.

The estimate of stroke incidence in patients diagnosed within 7 days from acute retinal artery occlusion varies 
significantly, ranging from $1 \%$ reported in a study based on administrative data, to $37 \%$ in a clinical study [3, 6-11]. This difference could be related to many variables, such as case definition and use of diagnostic imaging. A better understanding of this rate would be of great clinical relevance. It would help to address the issue of the need of urgent neurological evaluation, allowing a better management of the disease.

Therefore, we performed a systematic literature review and meta-analysis. The aim was to assess the incidence rate of acute cerebral ischaemia, based on magnetic resonance imaging (MRI), within 7 days from acute CRAO, BRAO or TMVL diagnosis. Asymptomatic stroke rate was investigated as a secondary outcome.

\section{Materials and methods}

The present study was conducted following the guidelines of the Preferred Report Items for Systematic Reviews and Meta-analyses (PRISMA) [12] and those of the meta-analysis of observational studies in epidemiology [13]. (PRISMA checklist: Table 1 in Supplementary information).

\section{Eligibility criteria for considering studies for this review}

All study design types except case reports were considered for inclusion in this meta-analysis. Only studies reporting the incidence rate of acute cerebral ischaemia diagnosed by diffusion-weighted magnetic resonance imaging (dw-MRI) within 7 days from the onset of CRAO, BRAO and TMVL were included.

Acute cerebral ischaemia on MRI was defined as presence of hyper-intense lesions on diffusion-weighted imaging (DWI), showing low signal on apparent diffusion coefficient maps $[14,15]$. Such acute lesions remain visible on DWI for only $7-14$ days $[15,16]$. The dw-MRI was the preferred diagnostic method of acute cerebral ischaemia due to its higher sensibility and specificity when compared with computed tomography scans in detecting early ischaemic brain lesions (97\% sensitivity and 100\% specificity vs. $47 \%$ and $93 \%$, respectively) $[14,17,18]$. Acute cerebral ischaemia could be symptomatic when other stroke-like symptoms were present, or asymptomatic when lacking additional neurological symptoms [9-11, 19]. This is clinically relevant as the presence of silent brain infarcts more than doubles the risk of subsequent stroke and dementia [19].

Only papers published in English and in peer-reviewed journals were included. No publication date or publication status restrictions were imposed. In cases where the same cohort of patients was included in more than one study, either the one with better quality or the most recent was included in our analysis.

The primary outcome measure was the rate of acute cerebral ischaemia on MRI within 7 days from acute MVL diagnosis in the CRAO, BRAO and TMVL cohorts, including both neurologically symptomatic and asymptomatic patients. The rate of asymptomatic acute cerebral ischaemia on MRI within 7 days from acute MVL diagnosis in each cohort was the secondary outcome measure.

\section{Search methods for identifying studies}

Studies were systematically searched for on Pubmed and Medline databases, and the Cochrane library, from inception to 30th January 2019. Four independent researchers (VB, PM, MF, AL) performed the electronic search according to the strategy shown in Table 2 in Supplementary information.

Reference lists of eligible studies were searched, along with meeting abstracts, reports from study registries that have not been published, and comments in reviews or other articles indicating the presence of "data not shown" relevant for the analysis.

\section{Study selection}

Two independent investigators (MF, AL) reviewed titles and abstracts of all studies found, applying eligibility criteria. All potentially eligible studies received a full-text evaluation to assess whether inclusion/exclusion criteria were completely satisfied. When eligibility assessment of a study required clarification or more information, the authors were contacted by email.

\section{Data collection and risk for bias assessment}

After study inclusion, two authors (MF, AL) proceeded to data extraction independently. When there were disagreements, consensus was achieved by involving a third investigator (AR). The following data were extracted from each included study: publication year, first author, location of the study, type of design, characteristics of each cohort including number of patients, gender, mean age, and race. The number of patients who experienced cerebral ischaemia, diagnosed by MRI, within 7 days from acute MVL, was extracted from each cohort, along with their characteristics (gender, race, and mean age). Similarly, data on asymptomatic cerebral ischaemia were collected.

The risk of bias for each included study was assessed by two investigators (PM, MR), relying on the Newcastle-Ottawa scale (NOS) [20]. A Newcastle-Ottawa score $\geq 6$ indicated a low-to-moderate risk of bias, as previously reported [21]. 


\section{Data synthesis and analysis}

Meta-analysis of the primary and secondary outcomes was performed. The pooled estimation of incidence of acute cerebral ischaemia was calculated as a proportion, along with $95 \%$ confidence intervals (CIs). Heterogeneity was evaluated by using the I [2] statistic, whose value indicated a moderate to high heterogeneity if $>50 \%$. The metaanalytic approach was based on a random effects model. A $P$ value was significant if $<0.05$. 'meta' packages within ' $R$ ' software, version 3.2.3., were used to perform the metaanalysis.

\section{Results}

\section{Study selection}

Figure 1 shows the study selection process. The electronic search identified a total of 7288 studies, of which 6871 were duplicates. Of the remaining 417, 23 received a full-text assessment to verify whether inclusion/exclusion criteria were met. Seventeen reports were ruled out, whereas six were included in the present study.

\section{Qualitative synthesis}

Table 1 displays the characteristics of the six included studies. A total of 335,133 and 126 patients affected by acute CRAO, BRAO and TMVL, respectively, were included. In all studies the diagnosis of acute cerebral ischaemia was based on dw-MRI. Brain imaging was performed within 7 days from the onset of MVL in all trials, with one reporting a mean time between MVL and MRI of $24 \mathrm{~h}$ [9]. Four out of the six included studies enrolled patients both with and without neurological symptoms related to the cerebral ischaemia. Otherwise, Golsari et al. [10] and Zhang et al. [11] included only patients with silent stroke in their trial and excluded symptomatic patients. There were five retrospective and one prospective study included in our analysis.

\section{Risk of bias}

All included studies showed a low-to-moderate risk of bias, since their score on the NOS was greater than 6 in all cases. In particular, all six reports scored 3 stars out of 4 in the selection and exposure categories, whereas all were given one star out of two in the comparability category.

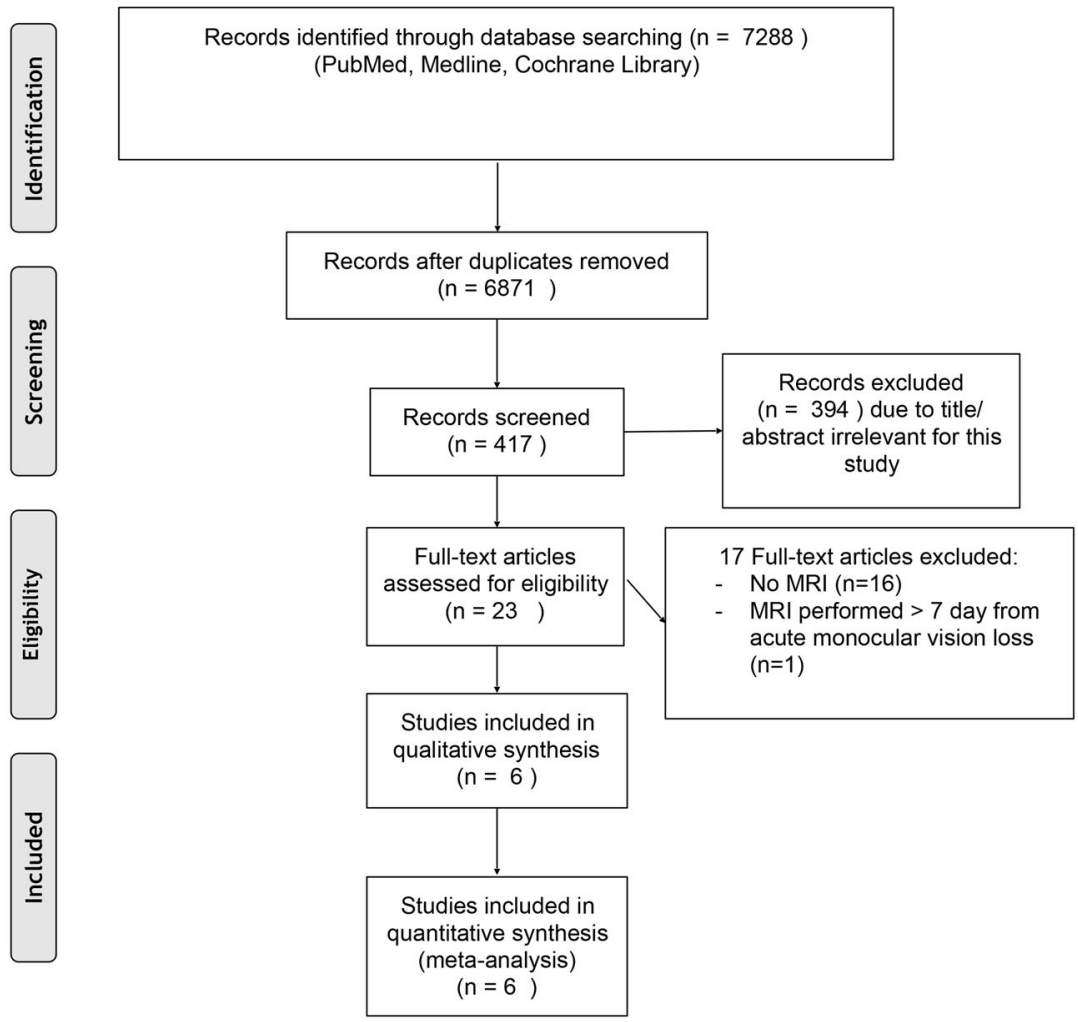




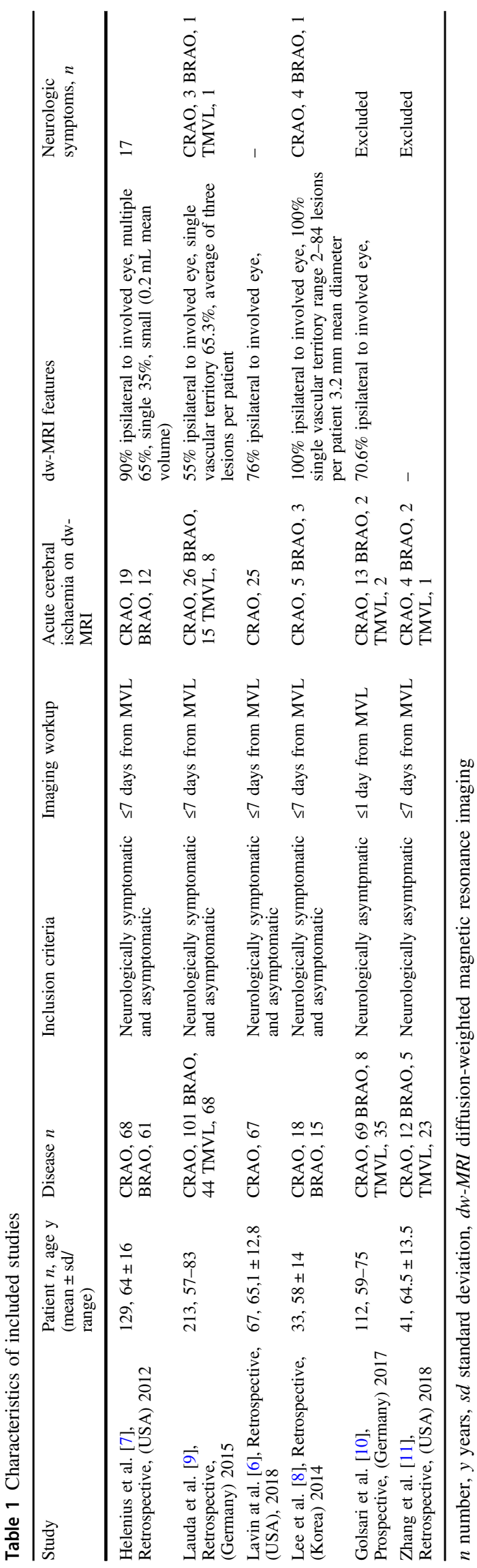

Pooled estimate of incidence of cerebral ischaemia on MRI

Meta-analysis of data from four studies was conducted to calculate the pooled estimate of incidence of cerebral ischaemia on MRI in the CRAO cohort. Overall, 75 patients out of 254 showed a positive brain imaging for acute cerebral ischaemia. According to the random effects model, the pooled proportion of cerebral ischaemia was 0.30 , with CI from 0.24 to 0.36 (Fig. 2). There was no evidence of statistical heterogeneity between the included studies $\left(I^{2}=0 \%\right.$; $P=0.44)$.

Pooled analysis of incidence of cerebral ischaemia on MRI in the BRAO cohort was performed on data from three studies. Overall, acute cerebral ischaemia on MRI was found in 30 out of 120 patients. The random effects model yielded a pooled proportion of 0.25 , with CI from 0.16 to 0.37 (Fig. 3). No evidence of statistical heterogeneity was shown among the included studies $\left(I^{2}=33 \% ; P=0.22\right)$.

No meta-analysis was conducted in the TMVL cohort because only one study was included. In this study, acute cerebral ischaemia on MRI was detected in 8 out of 68 patients (11.8\% of cases).

\section{Pooled estimate of incidence of asymptomatic cerebral ischaemia on MRI}

The pooled estimate of incidence of asymptomatic cerebral ischaemia on MRI in the CRAO cohort was computed from the data of four studies. Overall, 41 patients out of 193 were diagnosed with silent acute cerebral ischaemia on MRI. The random effects model provided a pooled proportion of 0.22 , with CI from 0.16 to 0.28 (Fig. 1 in Supplementary information). No statistical heterogeneity was found $\left(I^{2}=0 \%\right.$; $P=0.44)$.

Meta-analysis of data from four studies was performed for the pooled estimate of incidence of asymptomatic cerebral ischaemia on MRI in the BRAO cohort. Overall, silent acute cerebral ischaemia on MRI was shown in 20 out of 70 patients. According to the random effects model, the pooled proportion was 0.29 , with CI from 0.20 to 0.41 (Fig. 2 in Supplementary information). There was no evidence of statistical heterogeneity $\left(I^{2}=\right.$ $0 \% ; P=0.58)$.

Data from three studies were pooled to calculate an estimate of incidence of asymptomatic cerebral ischaemia on MRI in the TMVL cohort. Overall, 10 out of 125 patients presented silent acute cerebral ischaemia on brain imaging. The random effects model yielded a pooled proportion of 0.08 , with CI from 0.05 to 0.15 (Fig. 3 in Supplementary information). No statistical heterogeneity was shown $\left(I^{2}=0 \% ; P=0.56\right)$. 
Fig. 2 Pooled estimate of incidence of cerebral ischaemia on magnetic resonance imaging in CRAO cohort

\section{Study}

Helenius 2012

Lauda 2015

Lavin 2018

Lee 2014

Random effects mode Heterogeneity: $I^{2}=0 \%, \tau^{2}=0, p=0.44$
Events Total

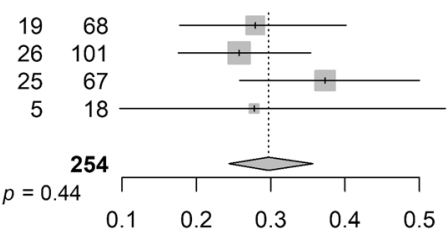

Proportion

$0.28[0.18 ; 0.40] 26.2 \%$ $0.26[0.18 ; 0.35] \quad 36.9 \%$ $0.37[0.26 ; 0.50] \quad 30.0 \%$ $0.28[0.10 ; 0.53] \quad 6.9 \%$ $0.30[0.24 ; 0.36] 100.0 \%$
Fig. 3 Pooled estimate of incidence of cerebral ischaemia on magnetic resonance imaging in BRAO cohort

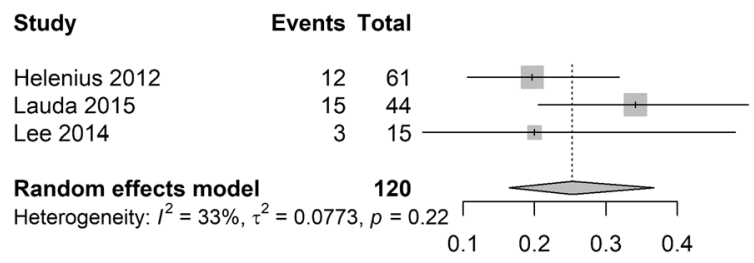

Study

Helenius 2012

Random effects mode Heterogeneity: $I^{2}=33 \%, \tau^{2}=0.0773, p=0.22 \quad 0.1 \quad 0.2 \quad 0.3 \quad 0.4$
Proportion $\quad 95 \%-\mathrm{Cl}$ Weight

$0.20[0.11 ; 0.32] \quad 42.0 \%$ $0.34[0.20 ; 0.50] \quad 42.6 \%$ $0.20[0.04 ; 0.48] 15.4 \%$

$0.25[0.16 ; 0.37] 100.0 \%$

\section{Discussion}

The present meta-analysis showed that $30 \%$ of patients with acute CRAO and $25 \%$ of patients with acute BRAO presented with acute cerebral ischaemia on MRI within 7 days from diagnosis. Such a high incidence supports the idea that patients with an acute retinal artery occlusion require urgent neurologic evaluation.

Clinical management of patients with acute retinal artery occlusion is still controversial.

On the one hand, Hayreh argued in his literature review that urgent neurologic evaluation in patients affected with acute CRAO without neurological symptoms is unnecessary and expensive [4]. In a previous study, the same author considered brain MRI in the absence of neurological symptoms was useless [22].

By sharp contrast, Arnold supported a different approach for acute CRAO, claiming the need of immediate referral to a stroke unit or ED to perform an urgent neurologic evaluation including brain imaging because of a high risk of acute ischaemic stroke, which could be asymptomatic [5]. Likewise, the very latest guidelines from the American Academy of Ophthalmology state that patients with acute CRAO have to be urgently sent to a stroke centre or ED and neuroimaging evaluation should be performed within $24 \mathrm{~h}$ [23].

However, clinical practice reports showed that urgent referral of CRAO to the emergency department has been carried out by $35 \%$ of ophthalmologists [24, 25] and more than $80 \%$ of retina specialists advised an outpatient workup for these patients [26].

The risk of stroke following retinal artery occlusion is well known, but the estimate of its incidence remains controversial [5]. Undoubtedly, a better understanding of the incidence of acute stroke within 7 days from diagnosis of ischaemic MVL, would reflect the clinical relevance of the issue and, as a consequence, would identify which could be the proper approach.
A previous meta-analysis conducted by Zhou et al. [27] reported a twofold higher risk of cerebrovascular diseases in patients with retinal artery occlusion compared with controls. The studies included in the Zhou et al. meta-analysis did not rely on neuroimaging for the diagnosis of cerebral ischaemia and the timeframe between retinal artery occlusion and stroke onset was not considered.

Two large population based studies confirmed a statistically significant increase in stroke risk after CRAO, in spite of different control groups and populations (South Korean vs. US Medicare population), with an incidence ranging between 1.2 and $2.2 \%$ during the first 7 days after CRAO [3, 28]. This lower brain ischaemia incidence when compared with our results may be explained by the following: (a) the two previous studies were based on administrative data-set reviews, therefore miscoding issues may have affected the results [29]; (b) the lack of dw-MRI in all patients with CRAO irrespective of symptoms did not allow the identification of silent ischaemia, thus possibly underestimating its incidence; (c) the lack of a control group in the studies included in the present meta-analysis might have been a source of bias, which in turn could have an influence on the results. However, an overestimation of the event seems unlikely given the great capability dw-MRI has to investigate early ischaemic injuries in the brain, with a sensitivity and specificity amounting to $88-100 \%$ and 95-100\%, respectively $[17,18]$.

Furthermore, up to $90 \%$ of patients with signs of acute cerebral infarction on MRI, performed within 7 days from MVL, did not suffer from any neurological symptoms [8]. This means that the largest percentage of ischaemic cerebral infarctions following MVL are clinically silent. For this reason, we investigated the pooled incidence of acute stroke in asymptomatic patients as a secondary outcome. Our findings showed that $21 \%$ of acute CRAO and $28 \%$ of acute BRAO patients were diagnosed with silent cerebral ischaemia on dw-MRI. The pooled incidence of silent stroke in the TMVL cohort was lower $(8 \%)$. This could be 
due to misdiagnosis of TMVL [8], and to its clinical features, which make TMVL an analogue of a transient ischaemic attack, rather than stroke [2].

The EXPRESS study [30] and SOS-TIA study [31] demonstrated that prompt evaluation and immediate treatment after transient ischaemic attack and minor stroke allowed the risk of recurrent stroke to be greatly reduced (by $80 \%$ ). Similarly, we suggest that patients with acute retinal artery occlusion require urgent brain imaging under the care of a neurologist. This management may decrease the risk of stroke recurrence and would obviously benefit both patient and the health care system, since the economic burden of urgent referral of these patients to the emergency department or stroke centre is outweighed by the economic and social costs produced by the disability and long-term care of stroke [32].

The following limitations apply to the present metaanalysis. First, few studies with relatively few patients were included. Only one study had a prospective design, while five out of six studies were retrospective. All studies were uncontrolled. The absence of a control group does not elucidate whether any variable might have had an influence on the results Consequently, bias (e.g., selection or misclassification bias) and confounding could have been introduced. In addition, only one study reported the mean time between acute retinal artery occlusion onset and MRI $(24 \mathrm{~h})$, whereas the others did not provide this information. Nonetheless, all included studies presented comparable methodologies, since all relied on dw-MRI and in all cases the timeframe between MVL diagnosis and MRI was $\leq 7$ days. In addition, the result of a meta-analysis has more value than a single cohort study. In particular, our metaanalysis yielded accurate results, given the narrow confidence interval and the absence of statistical heterogeneity. Finally, according to the NOS all included studies were characterized by a low publication bias.

In conclusion, this review supports prompt referral to the emergency department or stroke unit for neurological evaluation and brain imaging as part of the clinical management of acute retinal artery occlusion. This may identify concomitant strokes, which are mostly silent. A further randomized trial is needed to assess whether immediate initiation of treatment in those patients with asymptomatic cerebral ischaemia can reduce the incidence of stroke recurrence.

\section{Summary}

\section{What was known before?}

- The issue of urgent neurological assessment of patients with acute retinal artery occlusion has been debatable.
What this study adds?

- High rate of stroke in patients with retinal artery occlusion supports prompt referral to emergency department for neurological assessment.

Acknowledgements We are grateful to Dott. A. Bridgewood of the Scientific Bureau of the University of Catania for language editing.

\section{Compliance with ethical standards}

Conflict of interest The authors declare that they have no conflict of interest.

Publisher's note Springer Nature remains neutral with regard to jurisdictional claims in published maps and institutional affiliations.

\section{References}

1. Hayreh SS. Acute retinal arterial occlusive disorders. Prog Retin Eye Res. 2011;30:359-394.

2. Easton JD, Saver JL, Albers GW, Alberts MJ, Chaturvedi S, Feldmann E, et al. Definition and evaluation of transient ischemic attack: a scientific statement for healthcare professionals from the American Heart Association/American Stroke Association Stroke Council; Council on Cardiovascular Surgery and Anesthesia; Council on Cardiovascular Radiology and Intervention; Council on Cardiovascular Nursing; and the Interdisciplinary Council on Peripheral Vascular Disease. The American academy of neurology affirms the value of this statement as an educational tool for neurologists. Stroke. 2009;40:2276-2293.

3. Park SJ, Choi NK, Yang BR, Park KH, Lee J, Jung SY, et al. Risk and Risk Periods for Stroke and Acute Myocardial Infarction in Patients with Central Retinal Artery Occlusion. Ophthalmology. 2015;122:2336-2343.

4. Hayreh SS. Do patients with retinal artery occlusion need urgent neurolgical evaluation? Am J Ophthalmol. 2018. https://doi.org/ 10.1016/j.ajo.2018.08.015

5. Arnold AC. Urgent evaluation of the patient with acute central retinal artery occlusion. Am J Ophthalmol. 2018;196:xvi-xvii.

6. Lavin P, Patrylo M, Hollar M, Espaillat KB, Kirshner H, Schrag M. Stroke risk and risk factors in patients with central retinal artery occlusion. Am J Ophthalmol. 2018. https://doi.org/10.1016/ j.ajo.2018.08.027

7. Helenius J, Arsava EM, Goldstein JN, Cestari DM, Buonanno FS, Rosen BR, et al. Concurrent acute brain infarcts in patients with monocular visual loss. Ann Neurol. 2012;72:286-293.

8. Lee J, Kim SW, Lee SC, Kwon OW, Kim YD, Byeon SH. Cooccurrence of acute retinal artery occlusion and acute ischemic stroke: diffusion weighted magnetic resonance imaging study. Am J Ophthalmol. 2014;157:1231e1238.

9. Lauda F, Neugebauer H, Reiber L, Jüttler E. Acute silent brain infarction in monocular visual loss of ischemic origin. Cerebrovasc Dis. 2015;40:151e156.

10. Golsari A, Bittersohl D, Cheng B, Griem P, Beck C, Hassenstein A, et al. Silent brain infarctions and leukoaraiosis in patients with retinal ischemia: a prospective single-center observational study. Stroke. 2017;48:1392-1396.

11. Zhang LY, Zhang J, Kim RK, Matthews JL, Rudich DS, Greer $\mathrm{DM}$, et al. Risk of acute ischemic stroke in patients with 
monocular vision loss of vascular etiology. J Neuroophthalmol. 2018;38:328-333.

12. Liberati A, Altman DG, Tetzlaff J, Mulrow C, Gøtzsche PC, Ioannidis JP, et al. The PRISMA statement for reporting systematic reviews and meta-analyses of studies that evaluate healthcare interventions: explanation and elaboration. PLoS Med. 2009;6:e1000100.

13. Stroup DF, Berlin JA, Morton SC, Olkin I, Williamson GD, Rennie D, et al. Meta-analysis of observational studies in epidemiology: a proposal for reporting. Meta-analysis of observational studies in epidemiology (MOOSE) group. JAMA. 2000;283:2008-2012.

14. Davis DP, Robertson T, Imbesi SG. Diffusion-weighted magnetic resonance imaging versus computed tomography in the diagnosis of acute ischemic stroke. J Emerg Med. 2006;31:269-77. Review

15. Copen WA, Schwamm LH, Gonzàlez RG, Wu O, Harmath CB, Schaefer PW, et al. Ischemic stroke: effects of etiology and patient age on the time course of the core apparent diffusion coefficient. Radiology. 2001;221:27-34.

16. Burdette JH, Ricci PE, Petitti N, Elster AD. Cerebral infarction: time course of signal intensity changes on diffusion-weighted MR images. Am J Roentgenol. 1998;171:791-5.

17. Fiebach JB, Schellinger PD, Jansen O, Meyer M, Wilde P, Bender $\mathrm{J}$, et al. CT and diffusion weighted MR imaging in randomized order: diffusionweighted imaging results in higher accuracy and lower interrater variability in the diagnosis of hyperacute ischemic stroke. Stroke. 2002;33:2206-2210.

18. Gonzalez RG, Schaefer PW, Buonanno FS, Schwamm LH, Budzik RF, Rordorf G, et al. Diffusion weighted MR imaging: diagnostic accuracy in patients imaged within $6 \mathrm{~h}$ of stroke symptom onset. Radiology. 1999;210:155-162.

19. Vermeer SE, Longstreth WT Jr, Koudstaal PJ. Silent brain infarcts: a systematic review. Lancet Neurol. 2007;6:611-9.

20. Stang A. Critical evaluation of the Newcastle-Ottawa scale for the assessment of the quality of nonrandomized studies in metaanalyses. Eur J Epidemiol. 2010;25:603-605.

21. Fallico M, Russo A, Longo A, Pulvirenti A, Avitabile T, Bonfiglio $\mathrm{V}$, et al. Internal limiting membrane peeling versus no peeling during primary vitrectomy for rhegmatogenous retinal detachment: a systematic review and meta-analysis. PLoS ONE. 2018;13: e0201010.

22. Hayreh SS, Zimmerman MB. Ocular arterial occlusive disorders and carotid artery disease. Ophthalmol Retin. 2017;1:12-18.

23. Biousse V, Nahab F, Newman NJ. Management of acute retinal ischemia: follow the guidelines! Ophthalmology. 2018; 125:1597-1607.

24. Atkins E, Bruce BB, Newman NJ, Biousse V. Translation of clinical studies to clinical practice: survey on the treatment of central retinal artery occlusion. Am J Ophthalmol. 2009;148: $172 \mathrm{e} 173$

25. Youn TS, Lavin P, Patrylo M, Schindler J, Kirshner H, Greer DM, Schrag M. Current treatment of central retinal artery occlusion: a national survey. J Neurol. 2018;265:330-335.

26. Abel AS, Suresh S, Hussein HM, Carpenter AF, Montezuma SR, Lee MS. Practice patterns after acute embolic retinal artery occlusion. Asia Pac J Ophthalmol. 2017;6:37e39.

27. Zhou Y, Zhu W, Wang C. Relationship between retinal vascular occlusions and incident cerebrovascular diseases: A systematic review and meta-analysis. Medicine. 2016;95:e4075.

28. French DD, Margo CE, Greenberg PB. Ischemic stroke risk in medicare beneficiaries with central retinal artery occlusion: a retrospective cohort study. Ophthalmol Ther. 2018;7:125-131.

29. Goldstein LB. Accuracy of ICD-9-CM coding for the identification of patients with acute ischemic stroke. Effect of modifier codes. Stroke. 1998;29:1602-4.

30. Rothwell PM, Giles MF, Chandratheva A, Marquardt L, Geraghty O, Redgrave JN, et al. Early use of Existing Preventive Strategies for Stroke (EXPRESS) study. Effect of urgent treatment of transient ischaemic attack and minor stroke on early recurrent stroke (EXPRESS study): a prospective population-based sequential comparison. Lancet. 2007;370:1432e1442.

31. Lavallée PC, Meseguer E, Abboud H, Cabrejo L, Olivot JM, Simon O, et al. A transient ischaemic attack clinic with round-theclock access (SOSTIA): feasibility and effects. Lancet Neurol. 2007;6:953e 960 .

32. Mukundan G, Seidenwurm DJ. Economic and Societal Aspects of Stroke Management. Neuroimaging Clin N Am. 2018;28:683-689. 